E.C. HAMMARSTEN
${ }^{1}$
B. SZAPIRO
E. JANKOWSKA
J,3
J. FILEVICH
M.C. MARCONI
J.J. ROCCA $^{1,4}$

B. SZAPIRO ${ }^{1,2}$

E. JANKOWSKA ${ }^{1,3}$

M.C. MARCONI ${ }^{1,4}$

J.J. ROCCA ${ }^{1, \otimes}$

\section{Soft X-ray laser diagnostics of exploding aluminum wire plasmas}

\author{
${ }^{1}$ NSF ERC for Extreme Ultraviolet Science and Technology and Dept. of Electrical and Computer \\ Engineering, Colorado State University, Fort Collins, CO 80523, USA \\ ${ }^{2}$ Dept. of Physics, The University of the South, Sewanee, TN 37383, USA \\ ${ }^{3}$ Dept. of Physics, Wroclaw University of Technology, Wroclaw, Poland \\ ${ }^{4}$ Dept. of Physics, University of Buenos Aires, Argentina
}

Received: 27 Oktober 2003/Revised version: 13 January 2004 Published online: 7 April 2004 • ( ) Springer-Verlag 2004

ABSTRACT The evolution of current-driven thin single $\mathrm{Al}$ wire plasmas was studied with soft X-ray shadowgraphy and interferometry using a very compact capillary discharge-driven $\lambda=46.9 \mathrm{~nm}$ laser probe. Wires of $25 \mu \mathrm{m}$ diameter excited by current pulses with a $78 \mathrm{~A} / \mathrm{ns}$ increase rate were observed to expand uniformly at a rate of $3.5 \mu \mathrm{m} / \mathrm{ns}$. In contrast, an increase in the rate of energy deposited per unit mass was observed to give rise to significant early plasma instabilities. The results illustrate the use of table-top soft X-ray lasers as a new tool for the diagnostics of dense pulse power driven plasmas.

PACS 52.70.Kz; 52.59.Qy; 52.25.Vy; 52.50.Nr

\section{1}

\section{Introduction}

Interferometry with optical lasers has been used for decades to obtain multi-dimensional maps of the electron density in a large variety of plasmas [1]. However, free-free absorption and refraction of the probe beam limit the maximum electron density, plasma size, and plasma density gradient that can be probed [2]. More recently, laboratory-size soft $\mathrm{X}$-ray lasers have been used to extend the limits of plasma interferometry to larger plasma-scale lengths and higher plasma densities. A $15.5 \mathrm{~nm}$ Ne-like Y laser pumped by the Nova laser has successfully probed dense laser-created plasmas produced at Lawrence Livermore National Laboratory [2]. Due to limitations in the repetition rate of the X-ray laser, the experiments were limited to a few shots per day $[2,3]$. Recently, the use of compact saturated soft X-ray lasers [4-6] and the development of more robust interferometers [7] have advanced soft X-ray laser diagnostics as a practical tool for the study of high density plasmas [4-7]. Results of the study of the dynamics of several different laser-created plasmas have been recently reported [5-7]. Extremely compact capillary discharge soft-X-ray lasers $[8,9]$, which are portable and have spectral brightness similar to or higher than that of their laboratory-size predecessors, produce laser pulses of about $1 \mathrm{~ns}$ duration that are particularly well suited for the diagnostics of dense pulse-power driven plasmas. Herein we report the results of the study of the evolution of current-induced

Fax: +1-970/491-8671, E-mail: rocca@engr.colostate.edu explosions of thin wires using a capillary discharge $46.9 \mathrm{~nm}$ laser in combination with a Mach-Zehnder interferometer in which diffraction gratings are used as beam splitters.

The dynamics of thin exploding metal wire plasmas is of significant interest for the efficient generation of incoherent $X$-ray pulses of extremely high energy [10-12]. Fast $Z$-pinch implosions of cylindrical wire arrays have been shown to generate high energy density plasmas that emit very intense bursts of incoherent X-ray radiation capable of driving inertial confinement fusion targets [11]. In those experiments the main implosion phase, driven by multi-mega ampere currents, is preceded by a phase in which the heating, vaporization, and ionization of the wires in the array causes an expansion and merging of the individual plasmas into a continuous shell. This initial phase is driven by a pre-pulse with a current of the order of several $\mathrm{kA}$ per wire and rise times of the order of $100 \mathrm{~ns}$. Maximization of the X-ray yield requires an understanding of all phases of the plasma. The new success at Sandia National Laboratories Z-machine in the production of thermonuclear neutrons [11] driven by such X-ray burst further encourages the study of thin wire explosions.

Several recent studies of the dynamics of single wire explosions conducted utilizing hard X-ray shadowgraphy and optical laser techniques [13-22] have significantly increased the understanding of single wire explosions. The probing of exploding wire plasmas with soft X-ray lasers can potentially provide complementary information on the initial stages of the explosion, where the density is high. In the work reported in this paper a $46.9 \mathrm{~nm}$ laser probe was used to monitor the early stage of formation of the plasma and its subsequent expansion. The measurements show that the stability of the plasma depends on the excitation conditions, and that it deteriorates at high energy density deposition rates. The simultaneous analysis of the soft X-ray interferometry and absorption data suggests that the coronal plasma contains a significant concentration of desorbed low $Z$ impurities. The next section describes the experimental setup used to conduct the measurements, and Sect. 3 discusses the results of the study of $15 \mu \mathrm{m}$ and $25 \mu \mathrm{m}$ diameter exploding $\mathrm{Al}$ wires for different excitation conditions.

\section{Experimental setup}

The setup used to conduct the experiments consists of a table-top capillary discharge laser operating at 
$46.9 \mathrm{~nm}$ (SXRL), an amplitude division interferometer that uses diffraction gratings as beam splitters, and an exploding wire assembly. The probe beam was generated by a compact neon-like argon capillary discharge pumped laser operating in a single laser line at $46.9 \mathrm{~nm}[23,24]$. For this experiment laser pulse energies of $\sim 0.1 \mathrm{~mJ}$ and of $\sim 1.2 \mathrm{~ns}$ Full Width at Half Maximum (FWHM) duration were used. A beam divergence of $\sim 4.5 \mathrm{mrad}$ is typical of this laser. The characteristics of the soft X-ray laser are described in more detail in previous publications $[8,9,24]$. The short duration, high brightness, and very good spatial coherence [9] of the probing laser allows the generation of interferograms with a fringe visibility exceeding 50 percent over the entire field of view.

The diffraction grating interferometer (DGI) is based on a skewed Mach-Zehnder configuration schematically illustrated in Fig. 1a. The diffracted zero and first order beams from a diffraction grating form the two arms of the interferometer. The grating, ruled with a blaze angle of 1.33 degrees, is positioned at 79 degrees with respect to the soft X-ray laser beam to generate zero and first diffracted order beams of approximately equal intensities. Two elongated gold-coated mirrors are used to redirect the two beams onto the second grating, where they are recombined to create the interference pattern. As compared with interferometers based on thin film beam splitters this interferometer design has the advantages of a high throughput ( $\sim 6$ percent per arm) and high robustness. The interferometer was pre-aligned using a $\lambda=824 \mathrm{~nm}$ semiconductor laser having a temporal coherence length similar to that of the soft X-ray laser $(\sim 250 \mu \mathrm{m})$. To ensure that the $46.9 \mathrm{~nm}$ SXRL and the $824 \mathrm{~nm}$ semiconductor laser beams follow the same path, the diffraction gratings were ruled with two vertically separated regions of line densities 300 lines $/ \mathrm{mm}$ and 17.06 lines $/ \mathrm{mm}$ for the soft $\mathrm{X}$-ray and infrared laser beams respectively. The exploding wire was placed in the path of the zero order beam between the elongated mirror and the second diffraction grating. A flat relay mirror and a spherical mirror were used to image the plasma onto a gated charge-couple device/microchannel plate $(\mathrm{CCD} / \mathrm{MCP})$ detector with a magnification of $\sim 23 \mathrm{X}$. These mirrors were coated with $\mathrm{Si} / \mathrm{Sc}$ multilayer stacks to achieve a reflectivity of $\sim 40$ percent at the wavelength $46.9 \mathrm{~nm}$.

The exploding wire plasmas were generated by heating $99.99 \%$ pure $\mathrm{Al}$ wires of $15 \mu \mathrm{m}$ or $25 \mu \mathrm{m}$ diameter with current pulses of either $12.8 \mathrm{kA}$ or $18.3 \mathrm{kA}$ peak amplitude, corresponding to a current increase rate of $\sim 78 \mathrm{~A} / \mathrm{ns}$ or $\sim 110 \mathrm{~A} / \mathrm{ns}$ respectively. These current pulses were formed by discharging a $0.14 \mu \mathrm{F}$ capacitor charged to $14 \mathrm{kV}$ or $20 \mathrm{kV}$ through a triggered spark-gap switch, and delivered to the wire through a low inductance coaxial transmission line. A typical current pulse is shown in Fig. 1b. The different phases of the wire explosion as it is heated have been previously studied in correlation to the variations in the current and voltage pulses $[15,16]$. As the wire is heated its resistance increases, causing a drop in the rate of current increase. This is evident by the plateau seen in the current pulse shown in Fig. 1b. During this resistive heating phase the wire releases absorbed gases and, when sufficiently large temperatures are reached, metal vapor. When the released gasses and metal vapor break down in the presence of the fringing elec-

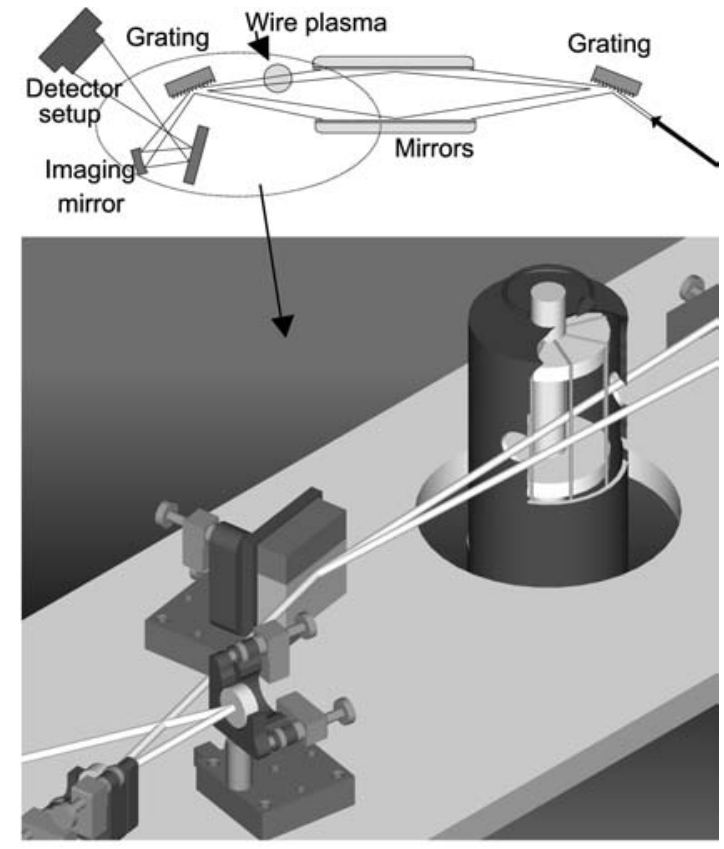

a

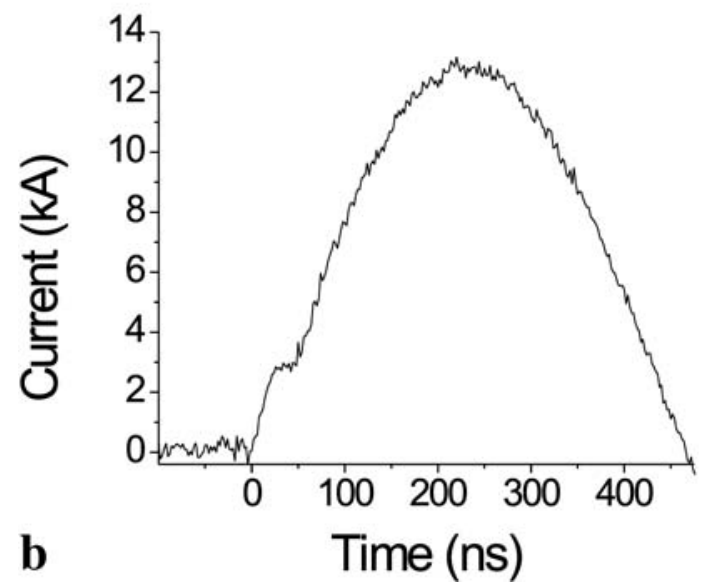

FIGURE 1 Experimental setup utilized in the study of exploding wire plasmas. a Schematic representation of the soft X-ray interferometer, with an enlarged representation of the wire assembly shown below. b Typical current pulse with peak amplitude of $12.8 \mathrm{kA}$

tric field, the current flowing through the wire core is effectively shunted through this coronal plasma, causing a rapid increase of the current. After this time most of the current flows through the coronal plasma and almost no additional energy is deposited in the wire core. Thus the majority of energy that contributes to the vaporization of the wire core is deposited before the end of the resistive heating phase [13]. A motorized wire holder, schematically shown in Fig. 1a, was used to allow for up to 8 single wire explosions to be performed without having to break vacuum. The wires were connected to a motorized brass disk that constitutes the top electrode. The lower ends of the wires were attached to $1 / 8$ th inch diameter stainless steel ball bearings that kept the wires vertically elongated, and which made electrical contact with a lower electrode to close the circuit for a single wire at a given time, allowing for single wire explosions. The top disc electrode was motorized to remotely position each wire along the path of the 
probe laser. A cylindrical metal shield was positioned around the wire array to minimize debris reaching the optics.

\section{Measurements and discussion}

Figure 2 shows a sequence of $46.9 \mathrm{~nm}$ shadowgrams/interferograms describing the early stages of the explosion of $25 \mu \mathrm{m}$ diameter $\mathrm{Al}$ wires excited by a current pulse with a current increase rate of $78 \mathrm{~A} / \mathrm{ns}(12.8 \mathrm{kA}$ peak amplitude with a $10 \%-90 \%$ rise time of $\sim 130 \mathrm{~ns})$. The timing relative to the start of the current pulse is specified. The reasonably good shot to shot reproducibility of these wire explosions allows the mapping of the evolution of the expanding wire by obtaining a sequence of images, each corresponding to a single wire explosion fired at different delays with respect to the probe laser pulse. The first frame of the sequence in Fig. 2 is a reference shot showing the $25 \mu \mathrm{m}$ wire shadow before firing the discharge. The onset of expansion of the absorption region illustrated in the subsequent frames is observed to occur during the resistive heating phase. For some shots the expansion is observed to start even before the current reaches its plateau. This suggests impurity atoms are released early from the surface of the wire as it is heated. Subsequent frames depict a rapid expansion of the fully absorbed region that occurs with an asymptotic velocity of $\sim 3.5 \mu \mathrm{m} / \mathrm{ns}$ [25] as indicated in Fig. 3. This value compares well with previous measurements of the velocity of expansion of $\mathrm{Al}$ wires of similar diameter obtained with hard X-ray radiographs and $532 \mathrm{~nm}$ shadowgraphy techniques [16,21]. During the onset of the recorded expansion, the central part of the vaporized wire, which contains a large density of neutral and singly ionized atoms that can be photo-ionized by the $26.5 \mathrm{eV}$ photons of the probe laser, completely absorbs the probe beam. In the surrounding outer shell the absorption decreases and a deflection of the interference fringes is observed. Later in time the probe beam is partially transmitted at all locations, as illustrated in Fig. 4, for a $25 \mu \mathrm{m}$ wire plasma exploded at a rate of $110 \mathrm{~A} / \mathrm{ns}, 131 \mathrm{~ns}$ after the initiation of the current pulse. The fact that at this time the probe beam is partially transmitted at all locations is an indication that the wire has been completely

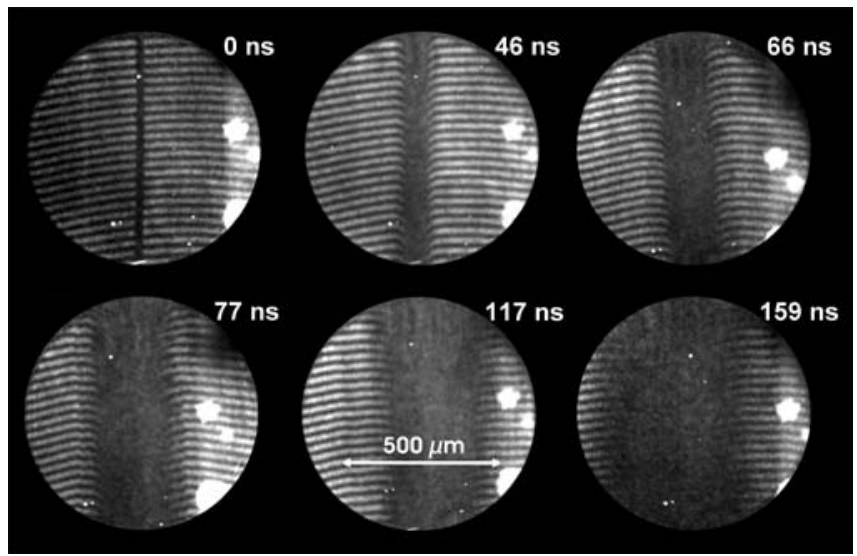

FIGURE 2 Sequence of interferograms/shadowgrams corresponding to $25 \mu \mathrm{m}$ current-driven $\mathrm{Al}$ wire explosions excited at a current increase rate of $78 \mathrm{~A} / \mathrm{ns}$. The time is measured with respect to the beginning of the current pulse

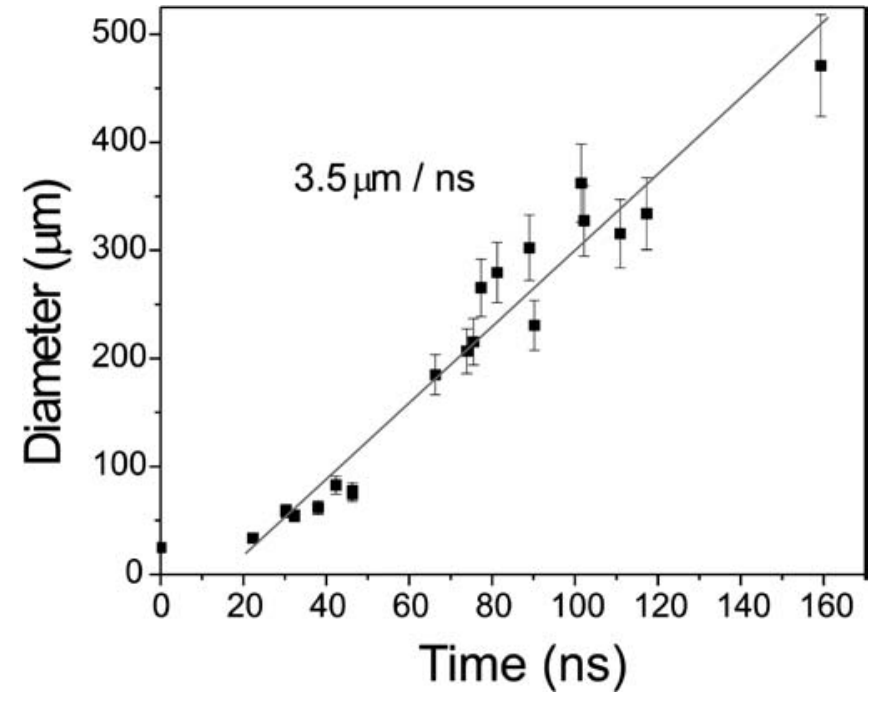

FIGURE 3 Measured evolution of the diameter of the soft X-ray absorbing region of the plasma corresponding to the explosion of $25 \mu \mathrm{m}$ diameter $\mathrm{Al}$ wires excited at a current increase rate of $78 \mathrm{~A} / \mathrm{ns}$. The expansion rate is $3.5 \mu \mathrm{m} / \mathrm{ns}$

vaporized, and that no fraction of the wire core remains in the solid or liquid phase.

The degree of uniformity of the explosion is of considerable interest. Significant instabilities have been detected in previous studies in wires explosions excited by high current pulses [26-28]. Schlieren images taken with a $694.3 \mu \mathrm{m}$ Ruby laser and absorption radiographs taken with $2-6 \mathrm{keV}$ $\mathrm{X}$-rays show the early onset of $m=0$ instabilities in $15 \mu \mathrm{m}$ Al wires exploded at a rate of $2.3 \mathrm{kA} / \mathrm{ns}(150 \mathrm{kA}, 65 \mathrm{~ns}$ $10 \%-90 \%$ rise time) [27]. The $m=0$ instability was also seen in Schlieren images of $100 \mu \mathrm{m}$ diameter $\mathrm{Al}$ wires excited with 100-350 kA peak current pulses, with FWHM of $100 \mathrm{~ns}$, taken with a $337 \mathrm{~nm}$ nitrogen laser [28]. In the present study conducted at significantly lower current, the plasma expan-

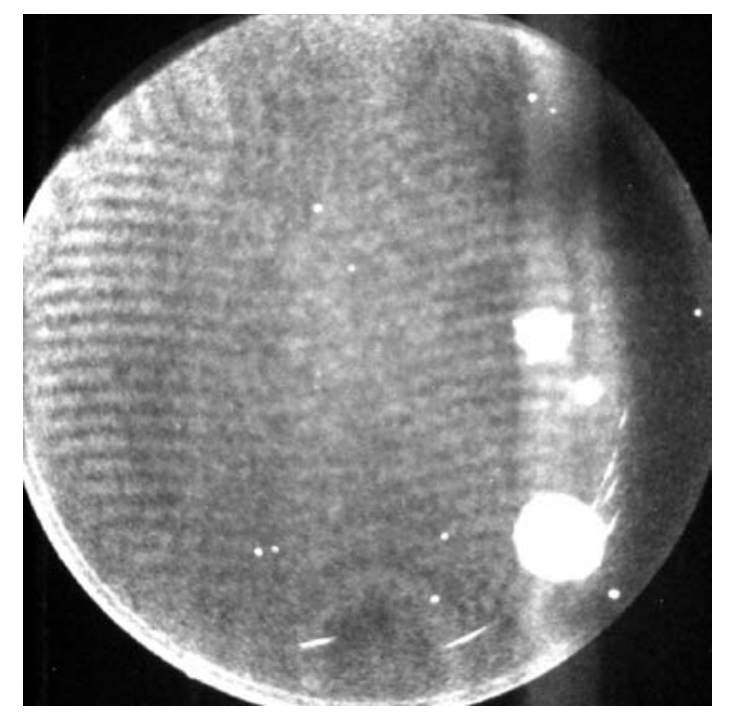

FIGURE 4 Interferogram of a $25 \mu \mathrm{m}$ diameter $\mathrm{Al}$ wire taken at $131 \mathrm{~ns}$ from the beginning of the current pulse. The transmission of the laser probe beam at all locations indicates that the metal core has been totally vaporized 
sion of $25 \mu \mathrm{m}$ diameter wires excited at the current increase rate of $78 \mathrm{~A} / \mathrm{ns}$ was observed to be uniform along the wire length for all times, indicating the absence of significant instabilities (Fig. 2). In contrast, a $50 \%$ increase in the rate of energy deposition was enough to give rise to significant instabilities. Interferograms corresponding to $25 \mu \mathrm{m}$ wires excited with an increased current rate of $110 \mathrm{~A} / \mathrm{ns}$ (current pulse amplitude $18.3 \mathrm{kA}$ and $10 \%-90 \%$ risetime of $\sim 130 \mathrm{~ns}$ ) often show the growth of significant non-uniformities very early in the evolution of the plasma, as illustrated in Fig. 5. Similar instabilities were also consistently observed when the current increase rate was maintained and the wire diameter was decreased. A sequence of shots corresponding to $15 \mu \mathrm{m}$ diameter $\mathrm{Al}$ wires excited at $78 \mathrm{~A} / \mathrm{ns}$ is shown in Fig. 6. In this case, the instability is observed to appear as early as $30 \mathrm{~ns}$ from the beginning of the current pulse and to rapidly grow in amplitude during the subsequent tens of nanoseconds.

The fringe shift observed in the coronal plasma region can be measured to obtain radial profiles of the index of re-

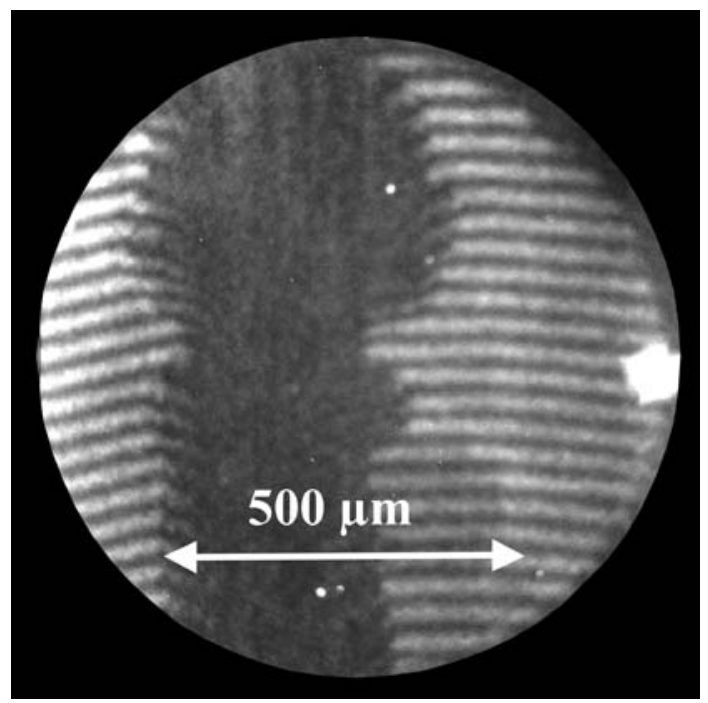

FIGURE 5 Interferogram/shadowgram of $25 \mu \mathrm{m}$ current-induced $\mathrm{Al}$ wire plasma excited at a current increase rate of $110 \mathrm{~A} / \mathrm{ns}$ showing instabilities. The image was taken $57 \mathrm{~ns}$ after the beginning of the current pulse

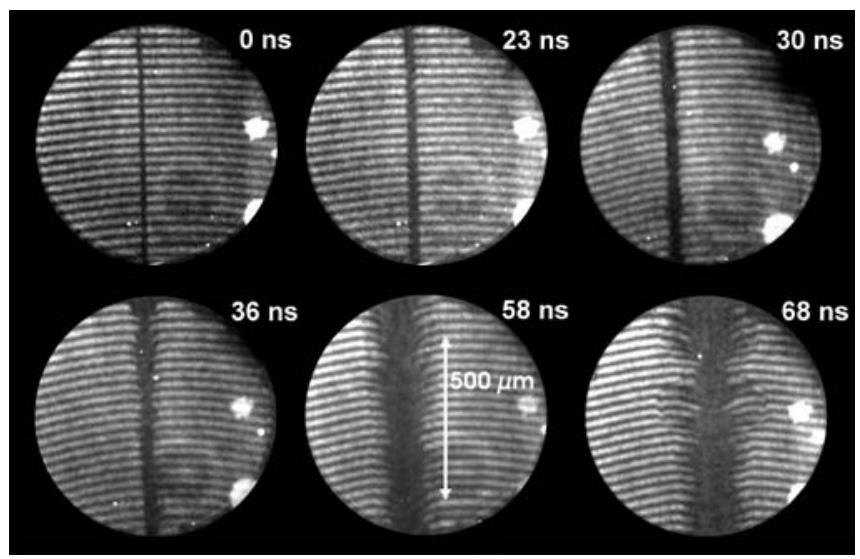

FIGURE 6 Series of interferograms/shadowgrams corresponding to $15 \mu \mathrm{m}$ $\mathrm{Al}$ wires excited at a current increase rate of $78 \mathrm{~A} / \mathrm{ns}$, showing the early onset of instabilities. The time is measured with respect to the beginning of the current pulse

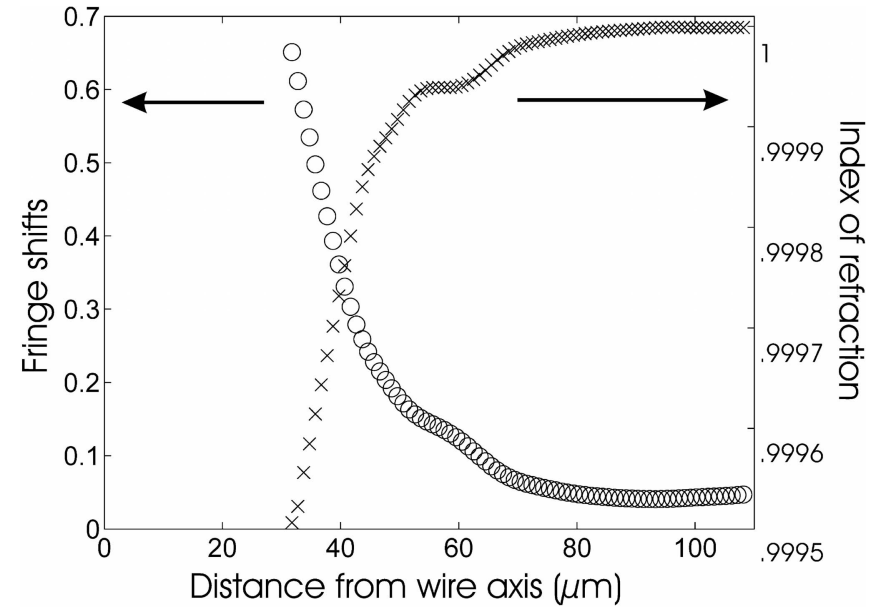

FIGURE 7 Measured fringe shift and corresponding radial index of refraction. The data corresponds to an interferogram of a $25 \mu \mathrm{m}$ diameter $\mathrm{Al}$ wire excited by a $78 \mathrm{~A} / \mathrm{ns}$ current pulse, at $46 \mathrm{~ns}$ from the beginning of the current pulse

fraction using an Abel inversion [29]. As an example, Fig. 7 shows the measured fringe shift and corresponding index of refraction as a function of the distance from the wire axis for the interferogram at $46 \mathrm{~ns}$ delay in Fig. 2, corresponding to the $25 \mu \mathrm{m}$ wire excited at a rate of $78 \mathrm{~A} / \mathrm{ns}$. Due to the short wavelength of the probe beam and the small dimension of the plasma, refraction effects can be neglected in this analysis. This was corroborated by computing the ray's trajectory based on the measured index of refraction distributions, which showed negligible deflection of the probe beam. However, the plasma density distribution can not be easily determined from the refractive index profile due to the fact that in relatively low temperature plasmas the refractive index can contain contributions not only from free electrons but also from the bound electrons of the neutral and ionized atoms.

The combined analysis of the fringe shift and absorption data could in principle provide a way to separate the contribution of bound electrons from that of free electrons. This approach is based on the fact that for a $46.9 \mathrm{~nm}$ probe the free-electron absorption is computed to be negligible in comparison with the absorption caused by the photoionization of $\mathrm{Al} \mathrm{I}$ and $\mathrm{Al} \mathrm{II}$ atoms (this holds true for a broad range of plasma parameters, including atom densities between $10^{19}$ and $10^{21} \mathrm{~cm}^{-3}$ with corresponding electron density values estimated assuming Saha equilibrium for temperatures between 0.1 and $10 \mathrm{eV}$ ). Therefore the density of Al I and Al II (Al III and higher ions do not contribute to the absorption) could be determined from the absorption data with knowledge of the photoionization cross-sections, and the result could subsequently be used in combination with the fringe shift data to calculate the electron density distribution. Such analysis, which would have to neglect the contribution of $\mathrm{Al}$ III ions to the fringe shift, is complicated by the lack of reliable scattering factors for the $\mathrm{Al}$ ions at this wavelength. Assuming this is a pure aluminum plasma dominated by neutral atoms, the analysis of the absorption data from the coronal plasma results in a density of atoms that would produce a fringe shift significantly larger than the measured one. If the plasma is dominated by $\mathrm{Al}$ II ions, agreement of the absorption and fringe shift data would occur if the value of the real component of the 
index of refraction of these ions is at least three times smaller than that of the neutral $\mathrm{Al}$ atoms. However the excessive absorption for the amount of observed fringe shift is likely to be due to the presence of a significant number of impurity atoms, such as oxygen and carbon which can be expected to be desorbed from the wire by the heating pulse. The comparison of the photoionization cross sections of $\mathrm{Al}$ atoms at $46.9 \mathrm{~nm}$ with that of $\mathrm{O}$ and $\mathrm{C}$ atoms shows that the latter are nearly an order of magnitude larger [30], suggesting that the presence of these impurities is the likely reason of our result. The indication that the coronal plasma might contain a significant density of impurity atoms corroborates recent studies which have arrived at a similar conclusion by analyzing the velocity of expansion of the plasma, or by the direct observation of line emission from impurities in the coronal plasma $[17,31]$.

\section{Conclusions}

We have used a table-top $46.9 \mathrm{~nm}$ laser to probe the early stages of the evolution of current-driven Al wire plasmas using shadowgraphy/interferometry techniques. The photoionization of the wire core atoms by the $26.5 \mathrm{eV}$ photons of the probe beam fully absorbs the laser beam during the early stages of the plasma evolution, limiting the region of visible fringe shifts to the surrounding coronal plasma. Sequences of images corresponding to $25 \mu \mathrm{m}$ diameter $\mathrm{Al}$ wires excited by a current increase rate of $78 \mathrm{~A} / \mathrm{ns}$ show an expanding plasma that is uniform along the wire length. The measured expansion velocity of $3.5 \mu \mathrm{m} / \mathrm{ns}$ is in good agreement with those reported from measurements that used other techniques $[13,14]$. In contrast, the expansion of $15 \mu \mathrm{m}$ diameter wires excited by a similar current increase rate, and that of $25 \mu \mathrm{m}$ diameter wires excited at a rate of $110 \mathrm{~A} / \mathrm{ns}$ are both observed to develop significant instabilities very early in the plasma evolution. The combined analysis of the fringe shift and absorption data suggests the coronal plasma might contain a significant concentration of desorbed impurities, which contribute to the absorption of the $26.5 \mathrm{eV}$ photons in the coronal region. The results illustrate the use of compact capillary discharge-driven soft X-ray lasers as a new tool for the diagnostics of dense pulse power driven plasmas.

ACKNOWLEDGEMENTS The authors would like to thank Jonathan Grava and Mike Purvis for their assistance with the experiments This work was supported by U.S. Department of Energy grant No. DE-FG0302NA00062. This work made use of Engineering Research Center Facilities supported by the National Science Foundation under award number EEC0310717. We also gratefully acknowledge the support of an equipment grant from the W.M. Keck Foundation.

\section{REFERENCES}

1 R.A. Alpher, D.R. White: Plasma Diagnostic Techniques, R.H. Huddlestone (Ed.), S.L. Leonard (Academic Press, NY 1965) pp. 431-76
2 L.B. Da Silva, T.W. Barbee Jr., R. Cauble, P. Celliers, D. Ciarlo, S. Libby, R.A. London, D. Matthews, S. Mrowka, J.C. Moreno, D. Ress, J.E. Trebes, A.S. Wan, F. Weber: Phys. Rev. Lett. 74, 3991 (1995)

3 A.S. Wan, T.W. Barbee, R. Cauble, P. Celliers, L.B. Da Silva, J.C. Moreno, P.W. Rambo, G.F. Stone, J.E. Trebes, F. Weber: Phys. Rev. E 55, 6293 (1997)

4 J.J. Rocca, C.H. Moreno, M.C. Marconi, K. Kanizay: Opt. Lett. 24, 420 (1999)

5 R.F. Smith, J. Dunn, J. Nilsen, V.N. Shlyaptsev, S. Moon, J. Filevich, J.J. Rocca, M.C. Marconi, J.R. Hunter, T.W. Barbee: Phys. Rev. Lett. 89, 065004 (2002)

6 J.J. Rocca, E.C. Hammarsten, E. Jankowska, J. Filevich, M.C. Marconi, S. Moon, V.N. Shlyaptsev: Phys. Plasmas 10, 2031 (2003)

7 J. Filevich, K. Kanizay, M.C. Marconi, J.L.A. Chilla, J.J. Rocca: Opt. Lett. 25, 356 (2000)

8 C.D. Macchietto, B.R. Benware, J.J. Rocca: Opt. Lett. 24, 1115 (1999)

9 Y. Liu, M. Seminario, F.G. Tomasel, C. Chang, J.J. Rocca, D.T. Attwood: Phys. Rev. A 63, 033802 (2001)

10 M.G. Haines, S.V. Lebedev, J.P. Chittenden, F.N. Beg, S.N. Bland, A.E. Dangor: Phys. Plasmas 7, 1672 (2000)

11 J.E. Bailey, G.A. Chandler, S.A. Slutz, G.R. Bennett, G. Cooper, J.S. Lash, S. Lazier, R. Lemke, T.J. Nash, D.S. Nielsen, T.C. Moore, C.L. Ruiz, D.G. Schroen, R. Smelser, J. Torres, R.A. Vesey: Phys. Rev. Lett. 89, 095004 (2002)

12 M.G. Haines: IEEE Trans. Plasma Sci. 26, 1275 (1998)

13 D.B. Sinars, M. Hu, K.M. Chandler, T.A. Shelkovenko, S.A. Pikuz, J.B. Greenly, D.A. Hammer, B.R. Kusse: Phys. Plasmas 8, 216 (2001)

14 D.B. Sinars, T.A. Shelkovenko, S.A. Pikuz, J.B. Greenly, D.A. Hammer: Phys. Plasmas 7, 1555 (2000)

15 P.U. Duselis, B.R. Kusse: Phys. Plasmas 10, 565 (2003)

16 K.M. Chandler, D.A. Hammer, D.B. Sinars, S.A. Pikuz, T.A. Shelkovenko: IEEE Trans. Plasma Sci. 30, 577 (2002)

17 P.U. Duselis, B.R. Kusse: AIP Conf. Proc. 651, 205 (2002)

18 G.S. Sarkisov, B.S. Bauer, J.S. De Groot: JETP Lett. 73, 69 (2001)

19 S.A. Pikuz, V.M. Romanova, N.V. Baryshnikov, M. Hu, B.R. Kusse, D.B. Sinars, T.A. Shelkovenko, D.A. Hammer: Rev. Sci. Instrum. 72, 1098 (2001)

20 S.A. Pikuz, T.A. Shelkovenko, D.B. Sinars, J.B. Greenly, Y.S. Dimant, D.A. Hammer: Phys. Rev. Lett. 83, 4313 (1999)

21 G.S. Sarkisov, S.E. Rosenthal, K.W. Struve, D.H. McDaniel, E.M. Waisman, P.V. Sasorov: AIP Conf. Proc. 651209 (2002)

22 S.A. Pikuz, T.A. Shelkovenko, A.R. Mingaleev, D.A. Hammer, H.P. Neves: Phys. Plasmas 6, 4272 (1999)

23 J.J. Rocca: Rev. Sci. Instrum. 70, 3799 (1999)

24 B.R. Benware, C.D. Macchietto, C.H. Moreno, J.J. Rocca: Phys. Rev. Lett. 81, 5804 (1998)

25 E. Jankowska, E.C. Hammarsten, B. Szapiro, J. Filevich, M.C. Marconi, J.J. Rocca: AIP Conf. Proc. 641, 498 (2002) A preliminary report of these results was made in a previous conf. proc. in which the current and current increase rates were stated incorrectly

26 A. Bartnik, G.V. Ivanenkov, L. Karpinski, A.R. Mingaleev, S.A. Pikuz, V.M. Romanova, W. Stepniewski, T.A. Shelkovenko, K. Jach: AIP Conf. Proc. 299, 449 (1993)

27 J.P. Chittenden, S.V. Lebedev, J. Ruiz-Camacho, F.N. Beg, S.N. Bland, C.A. Jennings, A.R. Bell, H.G. Haines, S.A. Pikuz, T.A. Shelkovenko, D.A. Hammer: Phys. Rev. E 61, 4370 (2000)

28 D.H. Kalantar, D.A. Hammer: Phys. Rev. Lett. 71, 3806 (1993)

29 K. Bockasten: J. Opt. Soc. Am. A 51, 943 (1961)

30 D.T. Attwood: Soft X-rays and extreme ultraviolet radiation: principles and applications (Cambridge Uni. Press, Cambridge, NY 2000)

31 G.S. Sarkisov, S.E. Rosenthal, K.W. Struve, D.H. McDaniel, E.M. Waisman, P.V. Sasorov: Presentation at 44th Annual Meeting Div. of Plasma Phys. (2002) 